\title{
Detention times of microswimmers close to surfaces: Influence of hydrodynamic interactions and noise
}

\author{
Konstantin Schaar ${ }^{1,2,3}$, Andreas Zöttl ${ }^{1}$, and Holger Stark ${ }^{1}$ \\ ${ }^{1}$ Institut für Theoretische Physik, Technische Universität Berlin, Hardenbergstrasse 36, 10623 Berlin, Germany \\ ${ }^{2}$ Institut für Theoretische Biologie, Humboldt Universität Berlin, Invalidenstrasse 43, 10115 Berlin, Germany and \\ ${ }^{3}$ Robert Koch-Institut, Seestrasse 10, 13353 Berlin, Germany
}

(Dated: October 16, 2018)

\begin{abstract}
After colliding with a surface, microswimmers reside there during the detention time. They accumulate and may form complex structures such as biofilms. We introduce a general framework to calculate the distribution of detention times using the method of first-passage times and study how rotational noise and hydrodynamic interactions influence the escape from a surface. We compare generic swimmer models to the simple active Brownian particle. While the respective detention times of source dipoles are smaller, the ones of pullers are larger by up to several orders of magnitude, and pushers show both trends. We apply our results to the more realistic squirmer model, for which we use lubrication theory, and validate them by simulations with multi-particle collision dynamics.
\end{abstract}

PACS numbers: 47.63.Gd, 47.63.mf, 87.10.Mn

Biological microswimmers such as bacteria are omnipresent in our everyday life. At the micron scale their locomotion in aqueous environment is determined by lowReynolds-number hydrodynamics and influenced by thermal and intrinsic biological noise [1, 2]. In real environments such as the human body [3] or the ocean [4, [5] microorganisms swim in the presence of soft or solid boundaries where they may form complex aggregates such as biofilms [6]. This letter develops a general approach for investigating the fundamental and biologically relevant question how long a swimming microorganism resides at bounding surfaces by accounting for both hydrodynamic swimmer-wall interactions and noise.

To develop an understanding for the accumulation and the dynamics of microorganisms near walls, several important aspects have been investigated recently: swimmer-wall hydrodynamic interactions 7 -10], thermal and intrinsic noise 7, 11], cilia- and flagella-wall interactions 12], bacterial tumbling 13], and buoyancy 14. Whether stochastic motion or swimmer-wall hydrodynamic interactions determine the reorientation of microswimmers at a surface and how they both influence the bacterial distribution between parallel plates has been discussed controversially [7, 8, 11]. Hydrodynamic interactions trap bacteria at surfaces [8, 15], force them to swim in circles [16], or even suppress bacterial tumbling [13]. However, non-tumbling bacteria 7, 11 or elongated artificial microswimmers [17] use rotational noise to escape from surfaces.

Artificial microswimmers such as active Janus particles or squirmers, which are driven by a surface velocity field, have been studied in front of a no-slip wall both in experiments [18, 19] and by theoretical models. The latter either include hydrodynamic interactions 15, 20 24 or only consider active Brownian particles [18, 25 28].

An important prerequisite for the observed accumulation near walls are the relatively large times microswim- mers reside at a surface before leaving it [17, 18]. In this article we call these swimmer-wall contact times detention times and calculate their distributions near a plane no-slip surface based on the method of first-passage times 29]. For generic microswimmers we demonstrate that hydrodynamic interactions, relative to pure rotational noise, can either increase the mean detention time by several orders of magnitude or also decrease it.

At low Reynolds number the motion of an axisymmetric microswimmer with orientation $\mathbf{e}$ in the presence of bounding surfaces is governed by the Langevin equations

$$
\begin{aligned}
& \dot{\mathbf{r}}=\mathbf{v}_{\mathrm{A}}+\mathbf{v}_{\mathrm{HI}}+\mathbf{v}_{\mathrm{N}}+\ldots, \\
& \dot{\mathbf{e}}=\boldsymbol{\Omega} \times \mathbf{e} \text { with } \boldsymbol{\Omega}=\boldsymbol{\Omega}_{\mathrm{HI}}+\boldsymbol{\Omega}_{\mathrm{N}}+\ldots,
\end{aligned}
$$

which account for the stochastic dynamics of position $\mathbf{r}$ and orientation e. Here we only consider the influence of the activity of the swimmer $\left(\mathbf{v}_{\mathrm{A}}=U \mathbf{e}\right.$ with bulk swimming velocity $U$ ), hydrodynamic interactions with the surface (HI), and noise (N). However, our approach can in principle be used for any dynamics which is of the form of Eqs. (10) and also include, e.g., steric or electrostatic interactions as well as external fluid flow.

We consider a spherical microswimmer, moving on a smooth trajectory, which reaches the wall at time $t_{0}$ with an angle $\theta_{0}$ against the surface normal (see Fig. 1 and a typical trajectory in the Supplemental Material [30]).
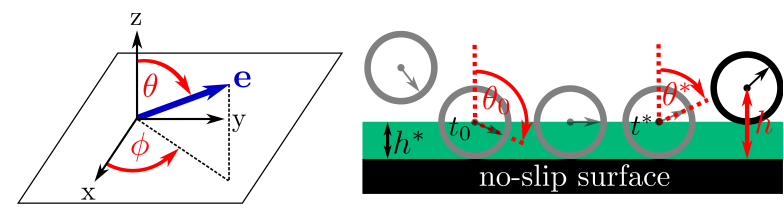

FIG. 1. Definition of coordinate system and sketch of a typical trajectory for a spherical microswimmer approaching a plane no-slip surface $\left(h=h^{*}\right)$ at time $t_{0}$ and leaving the surface at $t^{*}$. The detention time at the surface is $t^{*}-t_{0}$. 
This occurs at Péclet number $\mathrm{Pe}=U R / D_{t} \gg 1$, where $R$ is the radius and $D_{t}$ the translational diffusion coefficient of the swimmer. Typical values are $\mathrm{Pe} \gtrsim 10^{2}$ for bacteria, $\mathrm{Pe} \gtrsim 10^{3}$ for sperm cells and $\mathrm{Pe} \gtrsim 10^{4}$ for Chlamydomonas. The swimmer stays at a height $h \approx R$, so we neglect translational motion in the following [31]. The swimming direction e diffuses on the unit sphere but also drifts with angular velocity $\Omega_{\mathrm{HI}}=\Omega_{\mathrm{HI}} \mathbf{e}_{\phi}$. Once the swimming direction has reached the escape angle $\theta^{*}$, to be defined below for each swimmer type, the microswimmer leaves the surface at time $t^{*}$. This stochastic process is described by the Smoluchowski equation $\partial_{t} P=\mathcal{L} P=\left(-\mathcal{R} \cdot \boldsymbol{\Omega}_{\mathrm{HI}}+D_{r} \mathcal{R}^{2}\right) P$, where $\mathcal{R}=\mathbf{e} \times \boldsymbol{\nabla}_{\mathbf{e}}$ is the rotation operator and $D_{r}$ the rotational diffusion constant [26, 32].

Rotational diffusion along the azimuthal angle $\phi$ does not influence the escape from the surface and it is sufficient to consider the conditional probability $p\left(\theta, t^{*} \mid \theta_{0}, t_{0}\right)=\int_{0}^{2 \pi} \mathrm{d} \phi_{0} \int_{0}^{2 \pi} \mathrm{d} \phi P\left(\theta, \phi, t^{*} \mid \theta_{0}, \phi_{0}, t_{0}\right) . \quad$ To calculate the distribution of detention times at the surface, we use the Fokker-Planck approach of first-passage problems [29]. The integrated probability $g\left(\theta^{*}, t \mid \theta_{0}\right)=$ $\int_{\theta^{*}}^{\pi} p\left(\theta, t^{*} \mid \theta_{0}, t_{0}\right) \sin \theta \mathrm{d} \theta$ for finding the swimming direction in the angular interval $\left[\theta^{*}, \pi\right]$ at time $t=t^{*}-t_{0}$ obeys the adjoint Smoluchowski equation (see [30])

$$
\partial_{t} g\left(\theta^{*}, t \mid \theta_{0}\right)=\mathcal{L}^{+}\left(\theta_{0}\right) g\left(\theta^{*}, t \mid \theta_{0}\right)
$$

with $\mathcal{L}^{+}\left(\theta_{0}\right)=\Omega\left(\theta_{0}\right) \partial_{\theta_{0}}+D_{r} \partial_{\theta_{0}}^{2}$, where $\Omega\left(\theta_{0}\right)=$ $\Omega_{\mathrm{HI}}\left(\theta_{0}\right)+D_{r} \cot \theta_{0}$ is an effective angular drift velocity. To solve it, one uses at $\theta_{0}=\pi$ reflective $\left[\left.\partial_{\theta_{0}} g\left(\theta^{*}, t \mid \theta_{0}\right)\right|_{\pi}=0\right]$ and at $\theta_{0}=\theta^{*}$ absorbing $\left[g\left(\theta^{*}, t \mid \theta^{*}\right)=0\right]$ boundary conditions. Now, $-\partial_{t} g\left(\theta^{*}, t \mid \theta_{0}\right) \mathrm{d} t$ is the probability to leave the surface with escape angle $\theta^{*}$ at time $t$ in the time interval $\mathrm{d} t$, so

$$
f\left(\theta^{*}, t \mid \theta_{0}\right)=-\partial_{t} g\left(\theta^{*}, t \mid \theta_{0}\right)
$$

denotes the distribution of detention times $t=t^{*}-t_{0}$ for being trapped at the surface (DTD).

To investigate how hydrodynamic interactions compared to pure rotational noise influence the detention time, we calculate the DTD $f\left(\theta^{*}, t \mid \theta_{0}\right)$ for several model microswimmers by numerically solving Eq. (2) and using Eq. (3). From here on, we always rescale time by the ballistic time scale $\tau_{s}=R / U$ and introduce the persistence number $\mathrm{Pe}_{r}=\left(2 D_{r} \tau_{s}\right)^{-1}$. Since $\left(2 D_{r}\right)^{-1}$ is the orientational correlation time, $\mathrm{Pe}_{r} \gg 1$ means directed swimming 11, 33. Typical values are $\mathrm{Pe}_{r} \gtrsim 100$ for sperm cells [34] and non-tumbling E. coli [7], or $\mathrm{Pe}_{r} \approx 25$ for Chlamydomonas [7].

First, we consider a spherical active Brownian particle (ABP) with $\Omega_{\mathrm{HI}}=0$ near a surface 26,28 . The escape angle is simply $\theta^{*}=\pi / 2$. From the known propagator of free rotational diffusion [35], one can determine $g\left(\theta^{*}, t \mid \theta_{0}\right)$
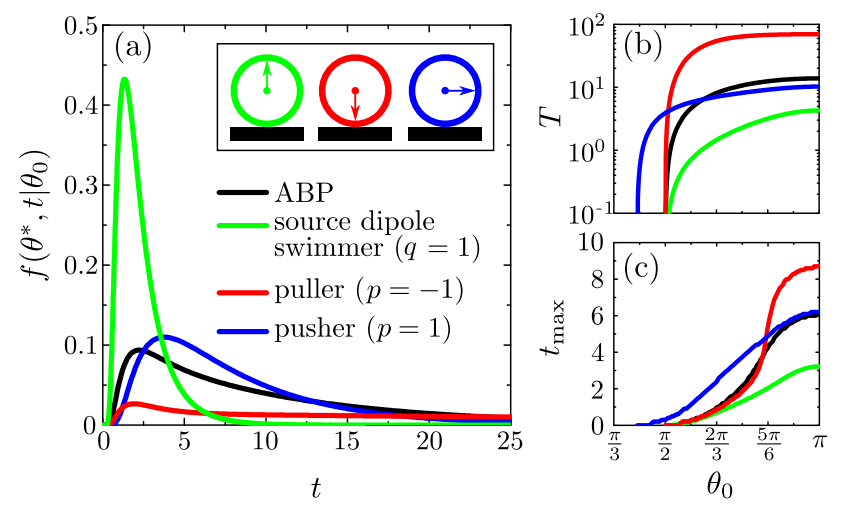

FIG. 2. (a) DTD for ABP and source- and force-dipole swimmer with $\mathrm{Pe}_{r}=10$ and an initial angle $\theta_{0}=3 \pi / 4$. (b) Mean detention time $T$ versus initial angle $\theta_{0}$. (c) Most likely detention time $t_{\max }$ (maximum of $f$ ).

and ultimately the DTD becomes

$$
\begin{aligned}
f\left(\frac{\pi}{2}, t \mid \theta_{0}\right) & =\frac{\pi}{2 \mathrm{Pe}_{r}} \sum_{l=1, \text { odd } l}^{\infty}(-1)^{\frac{l+1}{2}} e^{-l(l+1) t /\left(2 \mathrm{Pe}_{r}\right)} \\
& \times \frac{l(2 l+1)}{2^{l-1}}\left(\begin{array}{c}
l-1 \\
\frac{l-1}{2}
\end{array}\right) P_{l}\left(\cos \theta_{0}\right),
\end{aligned}
$$

where $P_{l}\left(\cos \theta_{0}\right)$ are Legendre polynomials. The DTD is plotted in Fig. 2(a) for $\theta_{0}=3 \pi / 4$ and $\mathrm{Pe}_{r}=10$. The mean detention time $T=\int_{0}^{\infty} t f\left(\theta^{*}, t \mid \theta_{0}\right) \mathrm{d} t$ of the ABP at the surface is calculated following Ref. [29],

$$
T^{\mathrm{ABP}}=2 \mathrm{Pe}_{r} \ln \left(1-\cos \theta_{0}\right) .
$$

We plot $T^{\mathrm{ABP}}$ versus $\theta_{0}$ in Fig. 2(b). Note that the most likely detention time $t_{\max }$ [see Fig. 2(c)] is much smaller compared to $T^{\mathrm{ABP}}$ due to the slow decay of $f\left(\theta^{*}, t \mid \theta_{0}\right)$.

Second, we consider microswimmers which generate either a force-dipole flow field of strength $p$ or a source dipole field of strength $q>0$ in the surrounding fluid [2]. Examples for the first case are pushers $(p>0)$ such as bacteria or pullers $(p<0)$ such as the biflagellated algae Chlamydomonas. Source dipoles are realized by active droplets [36] or Paramecia [37]. Each flow field is described by a flow singularity located in the center of the swimmer. For simplicity, we assume that the description by singularities is still valid close to the wall (see also the discussion in [7, 15]). Their flow fields interact hydrodynamically with the surface and thereby generate wallinduced angular velocities $\Omega_{\mathrm{HI}}$ of the microswimmers. At the wall $(h=R)$ they read $\Omega_{\mathrm{HI}}=3 p \sin \theta \cos \theta / 8$ for the force dipole and $\Omega_{\mathrm{HI}}=-3 q \sin \theta / 8$ for the source dipole, respectively [8, 15, 38]. The stable orientations $\theta_{s}$ of our swimmer types at the wall in the absence of noise are sketched in the inset of Fig. 2(a). They are calculated from $\Omega_{\mathrm{HI}}\left(\theta_{s}\right)=0$ and $\partial \Omega_{\mathrm{HI}}(\theta) /\left.\partial \theta\right|_{\theta=\theta_{s}}<0$.

Hydrodynamic interactions of the source dipole $(q>0)$ always rotate the swimmer away from the surface until 
it leaves the surface at $\theta^{*}=\pi / 2$. Hence, the width of the DTD is much narrower compared to the ABP [see Fig. 2(a)]. The mean detention time $T$ plotted in Fig. 2(b) is much smaller compared to $T^{\mathrm{ABP}}$ for all incoming angles $\theta_{0}$ due to $\Omega_{\mathrm{HI}} \propto-q$ and the most likely detention time $t_{\max }$ is comparable to $T$ [see Fig. 2(c)].

The puller $(p<0)$ is rotated towards the surface by hydrodynamic interactions if $\theta>\pi / 2$ and can only escape if angular noise drives it to $\theta<\theta^{*}=\pi / 2$. As a consequence, the DTD only has a weakly pronounced maximum and decays very slowly [see Fig. 2(a)]. Therefore, at $\mathrm{Pe}_{r}=10$ the mean detention time of the puller is by an order of magnitude larger than for the ABP. We note that for biological swimmers direct flagella-wall interactions can significantly influence the reorientation at the wall. For the puller algae Chlamydomonas $\Omega_{\text {steric }}>0$, which rotates the cell away from the surface [12] and strongly decreases the detention times compared to ABPs [30].

The situation of the pusher $(p>0)$ is more complex. Due to hydrodynamic interactions it has a stable orientation parallel to the wall $\left[\theta_{s}=\pi / 2\right.$, see inset of Fig. 2(a)]. Since, in addition, the wall-induced velocity $\mathbf{v}_{\text {HI }}\left(\theta_{s}\right)$ pushes it towards the wall, a noiseless pusher always swims at the wall [8] and $T \rightarrow \infty$. In the presence of noise the swimmer orientation fluctuates about its stable direction. The pusher stays trapped until the escape angle $\theta^{*}<\pi / 2$ is reached, where the total swimmer velocity starts to point away from the wall. Thus, the escape angle is determined by the condition $\left[\mathbf{v}_{\mathrm{A}}\left(\theta^{*}\right)+\mathbf{v}_{\mathrm{HI}}\left(\theta^{*}\right)\right] \cdot \mathbf{e}_{z}=$ 0 , which gives $\theta^{*}=\arccos \left[\left(-4+\sqrt{16+27 p^{2}}\right) /(9 p)\right]$ [7, 8].

Hydrodynamic interactions of the pusher with the surface can either enhance or reduce the detention time compared to an ABP. On the one hand, increasing $p \propto \Omega_{\mathrm{HI}}$ from zero reduces the time to reach the stable orientation and thus the time to get closer to the escape angle $\theta^{*}<\pi / 2$. This can reduce the mean detention time compared to ABPs for small $p$ as illustrated in Fig. 2(b). On the other hand, increasing $p$ further traps the orientation more strongly at $\theta_{s}=\pi / 2$ and also pushes $\theta^{*}$ more and more away from $\theta_{s}$. Since rotational diffusion has to compensate for both effects, the detention time increases.

Figure 3(a) gives an overview of the force-dipole swimmer by plotting $T / T^{\mathrm{ABP}}$ in a color code versus $\mathrm{Pe}_{r}$ and $p$. For negative $p$ the strong increase of $T$ beyond $T^{\mathrm{ABP}}$ with increasing $|p|$ is visible and also documented in the inset for two values of $\mathrm{Pe}_{r}$. For small positive $p$ and for $\mathrm{Pe}_{r} \gtrsim 5$ a clear minimum of $T$ develops as just discussed (see also the inset). In particular, in region I one finds $T<T^{\mathrm{ABP}}$. For example, for $\mathrm{Pe}_{r}=160$ the minimum at $p=0.4$ amounts to $T / T^{\mathrm{ABP}}=0.18$. Interestingly, this minimum occurs at a dipole strength comparable to the one estimated for E. coli bacteria [7].

In region II, $T$ grows to $10 T^{\mathrm{ABP}}$ or well beyond. The orientation of the pusher has time to equilibrate about $\theta_{s}=\pi / 2$ and then attempts to reach $\theta^{*}$ by rotational noise. Indeed, one can rewrite the effective ro-

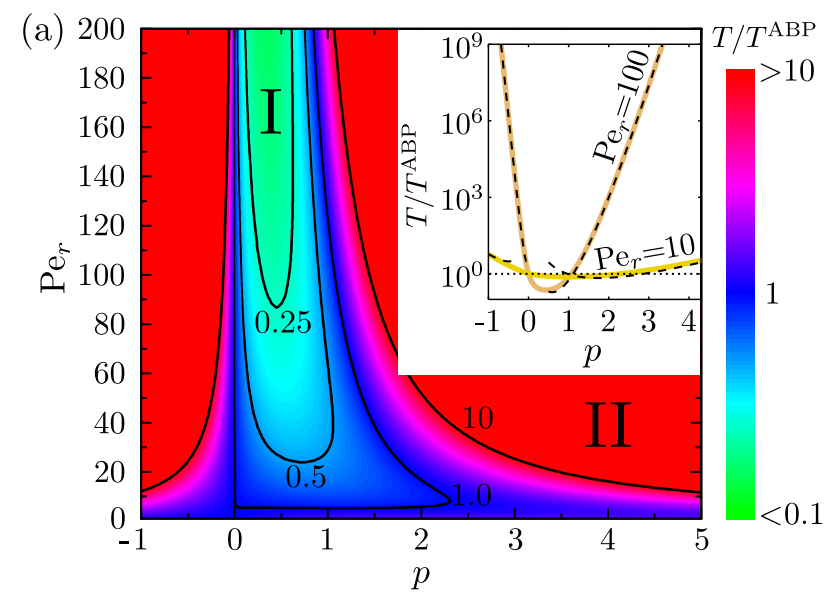

(b)

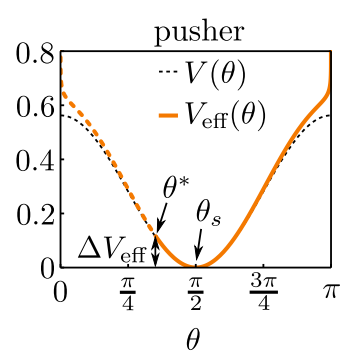

(c)

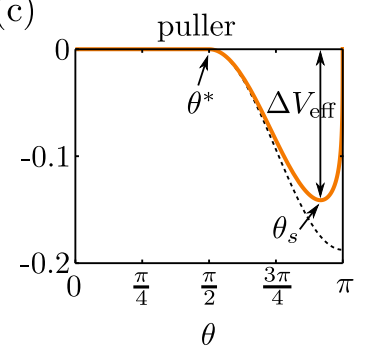

FIG. 3. (a) Mean detention time $T / T^{\mathrm{ABP}}$ for the force-dipole swimmer plotted versus $p$ and $\mathrm{Pe}_{r}$ for $\theta_{0}=3 \pi / 4$. Within region $\mathrm{I}, T / T^{\mathrm{ABP}}<1$, while in region $\mathrm{II}, T / T^{\mathrm{ABP}} \gg 1$. Inset: $T(p) / T^{\mathrm{ABP}}$ for two values of $\mathrm{Pe}_{r}$ and compared to Eqs. (6) and (7) (dashed lines). (b,c) Effective angular potentials $V_{\text {eff }}(\theta)$ and deterministic potentials $V(\theta)\left(\mathrm{Pe}_{r} \rightarrow \infty\right)$ for a pusher (b) $(p=3)$ and a puller (c) $(p=-1)$ at $\mathrm{Pe}_{r}=20$.

tational drift in Eq. (2) by introducing an effective angular potential $\Omega=-\partial V_{\text {eff }} / \partial \theta$ with $V_{\text {eff }}=V+V_{r}=$ $3 p \cos ^{2} \theta / 16-\ln (\sin \theta) /\left(2 \mathrm{Pe}_{r}\right)$, where the second term comes from the $3 \mathrm{D}$ rotational diffusion. However, the pusher escaping from the wall at $\theta^{*}$ cannot be viewed as a typical Kramers problem [29] since the orientation vector e does not pass a smooth potential barrier of height $\Delta V_{\text {eff }}$ when reaching the escape angle $\theta^{*}$. Instead, the swimmer orientation moves up the potential $V_{\text {eff }}$ by an amount $\Delta V_{\text {eff }}=V_{\text {eff }}\left(\theta^{*}\right)-V_{\text {eff }}\left(\theta_{s}\right)$ and when the pusher leaves the wall at $\theta^{*}$, it also leaves the range of $V_{\text {eff }}$ [see Fig. 3(b)]. However, we can derive an approximate formula for large $\mathrm{Pe}_{r} \Delta V_{\text {eff }}$ with the Arrhenius factor reminiscent of Kramers' mean escape time [30, 40],

$$
T^{\text {pusher }} \approx \frac{\sqrt{\pi}}{\left|V_{\mathrm{eff}}^{\prime}\left(\theta^{*}\right)\right| \sqrt{\mathrm{Pe}_{r} V_{\mathrm{eff}}^{\prime \prime}\left(\theta_{s}\right)}} e^{2 \mathrm{Pe}_{r} \Delta V_{\mathrm{eff}}}
$$

Interestingly, in case of the puller, the rotational-noise contribution $V_{r}$ shifts the most stable orientation to $\theta_{s}=$ $\pi-\arcsin \left[2 / \sqrt{-3 p \mathrm{Pe}_{r}}\right]<\pi$ [see Fig. 3](c)] 30 . Here, we can approximate $T$ by Kramers' formula [30, 41]

$$
T^{\text {puller }} \approx \frac{\pi}{\sqrt{\left|V_{\mathrm{eff}}^{\prime \prime}\left(\theta^{*}\right)\right| V_{\mathrm{eff}}^{\prime \prime}\left(\theta_{s}\right)}} e^{2 \mathrm{Pe}_{r} \Delta V_{\mathrm{eff}}} .
$$




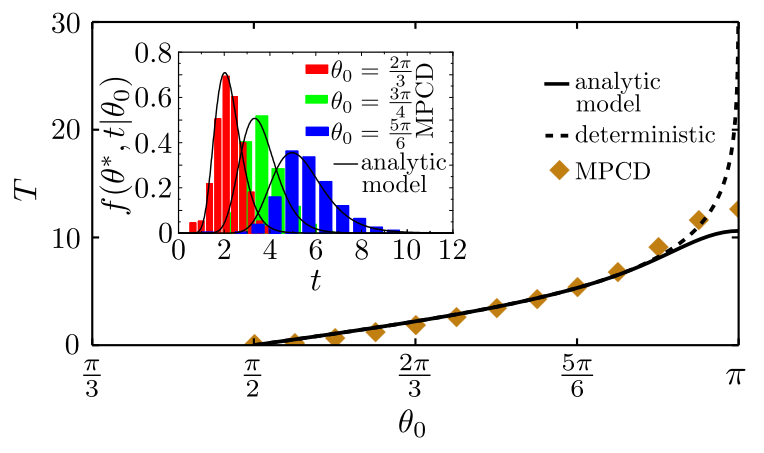

FIG. 4. Mean detention time $T$ of a neutral squirmer plotted versus the initial angle $\theta_{0}$ for $\mathrm{Pe}_{r}=110$ and $\epsilon=0.01$ (approximate mean distance from the wall measured from MPCD simulations) and compared to the analytic 1D model [Eqs. (2) and (3)], and the deterministic model $\left(\mathrm{Pe}_{r} \rightarrow \infty\right)$. Inset: Distribution of detention times from MPCD simulations and compared to analytic model.

The inset of Fig. 3(a) demonstrates that $T$ calculated from Eqs. (6) and (7) at $|p| \mathrm{Pe}_{r} \gg 1$ agrees very well with the one obtained by numerically solving Eqs. (2) and (3).

While so far we considered generic microswimmer models, we now turn to the spherical squirmer [42], which serves as a model for ciliated microorganisms such as Paramecium [37, 42] and Volvox [10] but also for active emulsion droplets [36]. The squirmer propels itself by an axisymmetric surface velocity field $\mathbf{v}_{s}=$ $\frac{3}{2}\left(1+\beta \mathbf{e} \cdot \hat{\mathbf{r}}_{s}\right)\left[\left(\mathbf{e} \cdot \hat{\mathbf{r}}_{s}\right) \hat{\mathbf{r}}_{s}-\mathbf{e}\right]$, where $\hat{\mathbf{r}}_{s}$ is the unit vector pointing from the center of the squirmer to its surface. The neutral squirmer $(\beta=0)$ creates the bulk flow field of a source dipole with $q=1 / 2$, while $\beta \neq 0$ adds an additional force-dipole field with $p=-3 \beta / 4$ [43]. Recent studies with squirmer-wall interactions already exist but without any noise [15, 22, 23, 44]. Using lubrication theory, the authors of Ref. [43] have calculated the dimensionless friction torque acting on the squirmer in front of a wall due to hydrodynamic interactions [43],

$$
M=(6 \pi / 5)(1-\beta \cos \theta) \sin \theta\left(\ln \epsilon^{-1}-c\right),
$$

where $\epsilon=h-1 \ll 1$ is a small distance and $c=$ const. This gives the wall-induced angular velocity $\Omega_{\mathrm{HI}}=-M / \gamma_{r}$, where $\gamma_{r}$ is the rotational friction coefficient near the surface [45, 46]. Note that the neutral squirmer $(\beta=0)$ behaves like the generic source dipole even close to the wall since $\Omega_{\mathrm{HI}} \sim-\sin \theta$. This might explain why farfield hydrodynamic interactions describe the near-wall swimming of neutral squirmers as shown in 15]. The $\beta$-dependent part in Eq. (8) adds to $\Omega_{\mathrm{HI}}$ the forcedipole term $\sim-p \sin \theta \cos \theta$. Acting alone, it rotates the squirmer pusher $(\beta<0)$ towards the wall and therefore it behaves like the generic puller with increased detention time and vice versa. These results are in accordance with recent simulations at finite Reynolds numbers 23].

To demonstrate that our 1D model is applicable, we perform full 3D mesoscale hydrodynamic simulations us- ing multi-particle collision dynamics (MPCD) 47 49]. It solves the Navier-Stokes equations for the fluid around the squirmer and the wall and naturally includes thermal fluctuations 50 53]. First, we numerically determine $c \approx 0.9[30$ ] and then explicitly simulate many swimming trajectories of swimmer-wall collision events for a neutral squirmer at different incoming angles. Figure 4 shows results for the mean detention time $T$ plotted versus the initial angle $\theta_{0}$, which agree well with our analytic model. The mean detention time of the deterministic swimmer, $T^{\text {det }} \propto \ln \tan \left(\theta_{0} / 2\right)$ 30], deviates from the full model only close to the unstable equilibrium orientation at $\theta=\pi$. Here $T^{\text {det }} \rightarrow \infty$, whereas noise renders $T$ finite and helps the swimmer to escape. The inset of Fig. 4 shows a convincing agreement of the DTDs determined from the analytic model and MPCD simulations.

To assess fluctuations of the position $h(t)$ above the surface, which influence $\Omega_{\mathrm{HI}}[8$, , 54], we may define an escape event by reaching a certain escape height $h^{*}>$ 1. For the state variable $\mathbf{y}(t)=(h, \theta)$ one defines the probability $g\left(\mathbf{y}^{*}, t \mid \mathbf{y}_{0}\right)$, for finding the swimmer below $h^{*}$ at time $t=t^{*}-t_{0}$ while the initial state $\mathbf{y}_{0}$ at $t_{0}$ starts at $h_{0} \in\left[1, h^{*}\right)$ and $\theta_{0} \in[0, \pi][55]$. The probability obeys the adjoint Fokker-Planck equation

$$
\begin{gathered}
\partial_{t} g\left(\mathbf{y}^{*}, t \mid \mathbf{y}_{0}\right)=\left[\left(\mathbf{v}_{\mathrm{A}}+\mathbf{v}_{\mathrm{HI}}\right) \cdot \mathbf{e}_{z} \partial_{h_{0}}+D_{t} \partial_{h_{0}}^{2}\right. \\
\left.-\left(\Omega_{\mathrm{HI}}+D_{r} \cot \theta_{0}\right) \partial_{\theta_{0}}+D_{r} \partial_{\theta_{0}}^{2}\right] g\left(\mathbf{y}^{*}, t \mid \mathbf{y}_{0}\right),
\end{gathered}
$$

with the initial condition $g\left(\mathbf{y}^{*}, t_{0} \mid \mathbf{y}_{0}\right)=\delta\left(\mathbf{y}^{*}-\mathbf{y}_{0}\right)$, and reflecting [at $\left.\mathbf{y}_{0}=(1, \pi)\right]$ and absorbing [at $\mathbf{y}_{0}=\left(h^{*}, \theta^{*}\right)$ ] boundary conditions for $g\left(\mathbf{y}^{*}, t \mid \mathbf{y}_{0}\right)$. Then, $f\left(\mathbf{y}^{*}, t \mid \mathbf{y}_{0}\right)=$ $-\partial_{t} g\left(\mathbf{y}^{*}, t \mid \mathbf{y}_{0}\right)$ is the DTD for detention time $t$. In [30] we show that for sufficiently large Pe and $h^{*}$ the detention times in the 2D model are larger compared to the 1D model. Small $h^{*}$ can also be reached by translational Brownian motion which reduces the detention times.

To conclude, based on the method of first-passage times, we developed a formalism to determine the distribution of detention times for microswimmers near a plane no-slip surface taking into account hydrodynamic interactions and rotational noise. For generic microswimmers such as source dipoles, pushers, and pullers we demonstrated that the mean detention time can vary over several orders of magnitude relative to the ABP depending on persistence number $\mathrm{Pe}_{r}$ and swimmer strengths $q, p$. This allows us to quantify the relative importance of hydrodynamic interactions and rotational noise. Our model also provides a route to quantify wall accumulation of microswimmer suspensions confined between two plates, as determined experimentally for different microorganisms [8, [1], 13, 56]. Our method can be extended to include further drift terms, for example, due to non-spherical swimmer shape, which further modifies the reorientation dynamics at the wall [11, 15]. Therefore, it offers a systematic approach for studying how artificial as well as biological microswimmers behave at surfaces. 
We thank Giovanni Volpe and Katrin Wolff for helpful discussions and the DFG for support within the research training group GRK 1558, within the priority program SPP 1726 "Microswimmers" (STA 352/11), and by grant STA 352/10-1.

[1] H. C. Berg, Random Walks in Biology (Princeton University Press, Princeton, NJ, 1993).

[2] E. Lauga and T. R. Powers, The hydrodynamics of swimming microorganisms, Rep. Prog. Phys. 72, 096601 (2009).

[3] D. Bray, Cell Movements (Garland Publishing, New York, 2000).

[4] M. E. Callow and J. A. Callow, Marine biofouling: a sticky problem, Biologist 49, 1 (2002).

[5] E. Rosenberg, O. Koren, L. Reshef, R. Efrony, and I. Zilber-Rosenberg, The role of microorganisms in coral health, disease and evolution, Nat. Rev. Microbiol. 5, 356 (2007).

[6] P. Watnick and R. Kolter, Biofilm, City of Microbes, J. Bacteriol. 182, 2675 (2000).

[7] K. Drescher, J. Dunkel, L. H. Cisneros, S. Ganguly and R. E. Goldstein, Fluid dynamics and noise in bacterial cell-cell and cell-surface scattering, Proc. Natl. Acad. Sci. U.S.A. 108, 10940 (2011).

[8] A. P. Berke, L. Turner, H. C. Berg, and E. Lauga, Hydrodynamic Attraction of Swimming Microorganisms by Surfaces, Phys. Rev. Lett. 101, 038102 (2008).

[9] E. Lauga, W. R. DiLuzio, G. M. Whitesides, and H. Stone, Swimming in Circles: Motion of Bacteria near Solid Boundaries, Biophys. J. 90, 400 (2006).

[10] K. Drescher, K. C. Leptos, I. Tuval, T. Ishikawa, T. J. Pedley, and R. E. Goldstein, Dancing Volvox: Hydrodynamic Bound States of Swimming Algae, Phys. Rev. Lett. 102, 168101 (2009).

[11] G. Li and J. X. Tang, Accumulation of Microswimmers near a Surface Mediated by Collision and Rotational Brownian Motion, Phys. Rev. Lett. 103, 078101 (2009).

[12] V. Kantsler, J. Dunkel, M. Polin, and R. E. Goldstein, Ciliary contact interactions dominate surface scattering of swimming eukaryotes, Proc. Natl. Acad. Sci. U.S.A. 110, 1187 (2013).

[13] M. M. Molaei, M. Barry, R. Stocker, and J. Sheng, Failed Escape: Solid Surfaces Prevent Tumbling of Escherichia coli, Phys. Rev. Lett. 113, 068103 (2014).

[14] I. Jung, K. Guevorkian, and J. M. Valles, Trapping of Swimming Microorganisms at Lower Surfaces by Increasing Buoyancy, Phys. Rev. Lett. 113, 218101 (2014).

[15] S. E. Spagnolie and E. Lauga, Hydrodynamics of selfpropulsion near a boundary: predictions and accuracy of far-field approximations, J. Fluid Mech. 700, 105 (2012).

[16] W. R. DiLuzio, L. Turner, M. Mayer, P. Garstecki, D. W. Weibel, H. C. Berg, and G. M. Whitesides, Escherichia coli swim on the right-hand side, Nature 435, 1271 (2005).

[17] D. Takagi, J. Palacci, A. B. Braunschweig, M. Shelley, and J. Zhang, Hydrodynamic capture of microswimmers into sphere-bound orbits, Soft Matter 10, 1784 (2014); S. E. Spagnolie, G. R. Moreno-Flores, D. Bartolo, and E. Lauga, Geometric capture and escape of a microswim- mer colliding with an obstacle, to be published in Soft Matter (2015).

[18] G. Volpe, I. Buttinoni, D. Vogt, H.-J. Kümmerer, and C. Bechinger, Microswimmers in patterned environments, Soft Matter 7, 8810 (2011).

[19] C. Kreuter, U. Siems, P. Nielaba, P. Leiderer, and A. Erbe, Transport phenomena and dynamics of externally and self-propelled colloids in confined geometry, Eur. Phys. J. Special Topics 222, 2923 (2013).

[20] D. G. Crowdy and Y. Or, Two-dimensional point singularity model of a low-Reynolds-number swimmer near a wall, Phys. Rev. E 81, 036313 (2010).

[21] D. G. Crowdy, Wall effects on self-diffusiophoretic Janus particles: a theoretical study, J. Fluid Mech. 735, 473 (2013).

[22] K. Ishimoto and E. A. Gaffney, Squirmer dynamics near a boundary, Phys. Rev. E 88, 062702 (2013).

[23] G.-J. Li and A. M. Ardekani, Hydrodynamic interaction of microswimmers near a wall, Phys. Rev. E 90, 013010 (2014).

[24] W. E. Uspal, M. N. Popescu, S. Dietrich, and M. Tasinkevych, Self-propulsion of a catalytically active particle near a planar wall: from reflection to sliding and hovering, Soft Matter 11, 434 (2015).

[25] S. van Teeffelen and H. Löwen, Dynamics of a Brownian circle swimmer, Phys. Rev. E 78, 020101(R) (2008).

[26] M. Enculescu and H. Stark, Active Colloidal Suspensions Exhibit Polar Order under Gravity, Phys. Rev. Lett. 107, 058301 (2011).

[27] C. F. Lee, Active particles under confinement: aggregation at the wall and gradient formation inside a channel, New J. Phys. 15, 055007 (2013).

[28] J. Elgeti and G. Gompper, Wall accumulation of selfpropelled spheres, Europhys. Lett. (EPL) 101, 48003 (2013).

[29] J. Honerkamp, Stochastic Dynamical Systems (VCH, New York, 1994), Chap. 6 and 9.

[30] See Supplemental Material at ... for additional information.

[31] The escape from a surface is indeed mainly determined by rotational motion, see [30].

[32] K. Wolff, A. M. Hahn, and H. Stark, Sedimentation and polar order of active bottom-heavy particles, Eur. Phys. J. E 36, 43 (2013).

[33] J. Taktikos, V. Zaburdaev, and H. Stark, Collective dynamics of model microorganisms with chemotactic signaling, Phys. Rev. E 85, 051901 (2012).

[34] B. M. Friedrich and F. Jülicher, Steering Chiral Swimmers along Noisy Helical Paths, Phys. Rev. Lett. 103, 068102 (2009).

[35] B. J. Berne and R. Pecora, Dynamic Light Scattering (Dover, New York, 2000), Chap. 7.

[36] S. Thutupalli, R. Seemann, and S. Herminghaus, Swarming behavior of simple model squirmers, New J. Phys. 13, 073021 (2011).

[37] T. Ishikawa and M. Hota, Interaction of two swimming Paramecia, J. Exp. Biol. 209, 4452 (2006).

[38] We note that swimmer flow fields and hence $\Omega_{\mathrm{HI}}$ can be time-dependent [39] and may influence the DTDs, which we neglect in the following.

[39] K. Drescher, R. E. Goldstein, N. Michel, M. Polin, and I. Tuval, Direct Measurement of the Flow Field around Swimming Microorganisms, Phys. Rev. Lett. 105, 168101 (2010); J. S. Guasto, K. A. Johnson, and 
J. P. Gollub, Oscillatory Flows Induced by Microorganisms Swimming in Two Dimensions, ibid. 105, 168102 (2010).

[40] For an alternative, heuristic expression based on Kramers theory see Ref. 7].

[41] See [30] for the limit $|p| \mathrm{Pe}_{r} \rightarrow \infty$.

[42] J. Lighthill, On the Squirming Motion of Nearly Spherical Deformable Bodies through Liquids at Very Small Reynolds Numbers, Commun. Pure Appl. Math. 5, 109 (1952); J. R. Blake, A spherical envelope approach to ciliary propulsion, J. Fluid Mech. 46, 199 (1971).

[43] T. Ishikawa, M. P. Simmonds, and T. J. Pedley, Hydrodynamic interaction of two swimming model microorganisms, J. Fluid Mech. 568, 119 (2006).

[44] I. Llopis and I. Pagonabarraga, Hydrodynamic interactions in squirmer motion: Swimming with a neighbour and close to a wall, J. Non-Newtonian. Fluid Mech. 165, 946 (2010).

[45] B. Cichocki and R. B. Jones, Image representation of a spherical particle near a hard wall, Physica A 258, 273 (1998).

[46] We neglect here the small translational-rotational coupling.

[47] A. Malevanets and R. Kapral, Mesoscopic model for solvent dynamics, J. Chem. Phys. 110, 8605 (1999).

[48] R. Kapral, Multiparticle collision dynamics: simulation of complex systems on mesoscales, Adv. Chem. Phys. 140, 89 (2008).

[49] G. Gompper, T. Ihle, D. M. Kroll, and R. G. Winkler, Multi-Particle Collision Dynamics: A Particle-Based Mesoscale Simulation Approach to the Hydrodynamics of Complex Fluids, Adv. Polym. Sci. 221, 1 (2009).

[50] M. T. Downton and H. Stark, Simulation of a model microswimmer, J. Phys. Condens. Matt. 21, 204101 (2009).

[51] I. O. Götze and G. Gompper, Mesoscale simulations of hydrodynamic squirmer interactions, Phys. Rev. E 82, 041921 (2010).

[52] A. Zöttl and H. Stark, Nonlinear Dynamics of a Microswimmer in Poiseuille Flow, Phys. Rev. Lett. 108, 218104 (2012).

[53] A. Zöttl and H. Stark, Hydrodynamics Determines Collective Motion and Phase Behavior of Active Colloids in Quasi-Two-Dimensional Confinement, Phys. Rev. Lett. 112, 118101 (2014).

[54] G. Li, L.-K. Tam, and J. X. Tang, Amplified effect of Brownian motion in bacterial near-surface swimming, Proc. Natl. Acad. Sci. U.S.A. 105, 18355 (2008).

[55] K. Schaar, Hydrodynamics and Noise in MicroswimmerSurface Interactions, master thesis, Technische Universität Berlin, 2013.

[56] L. Rothschild, Non-random distribution of bull spermatozoa in a drop of sperm suspension, Nature 198, 1221 (1963).

[57] S. Redner, A Guide to First-Passage Processes (Cambridge University Press, New York, 2001), Chap. 2. 


\section{SUPPLEMENTAL MATERIAL}

\section{Adjoint Smoluchowski equation}

The dynamics of the conditional probability density $P\left(\mathbf{e}, t^{*} \mid \mathbf{e}_{0}, t_{0}\right)=P\left(\theta, \phi, t^{*} \mid \theta_{0}, \phi_{0}, t_{0}\right)$ for finding a swimmer in the orientation interval $[\mathbf{e}, \mathbf{e}+\mathrm{de}]$ at time $t^{*}$, if it was at orientation $\mathbf{e}_{0}$ at time $t_{0}$, is governed by the Smoluchowski equation

$$
\partial_{t^{*}} P\left(\mathbf{e}, t^{*} \mid \mathbf{e}_{0}, t_{0}\right)=\mathcal{L}(\mathbf{e}) P\left(\mathbf{e}, t^{*} \mid \mathbf{e}_{0}, t_{0}\right),
$$

where

$$
\mathcal{L}(\mathbf{e})=-\mathcal{R}(\mathbf{e}) \cdot \boldsymbol{\Omega}_{\mathrm{HI}}(\mathbf{e})+D_{r} \mathcal{R}^{2}(\mathbf{e})
$$

is the Smoluchowski operator and $\mathcal{R}(\mathbf{e})=\mathbf{e} \times \nabla_{\mathbf{e}}$ the rotation operator (see main text). Eq. (10) describes the forward evolution of the probability density $P\left(\mathbf{e}, t^{*} \mid \mathbf{e}_{0}, t_{0}\right)$ as a function of $\mathbf{e}$ and $t^{*}$ for the initial condition $P\left(\mathbf{e}, t_{0} \mid \mathbf{e}_{0}, t_{0}\right)=\delta\left(\mathbf{e}-\mathbf{e}_{0}\right)$ at $t^{*}=t_{0}$. One can also formulate the backward evolution of $P\left(\mathbf{e}, t^{*} \mid \mathbf{e}_{0}, t_{0}\right)$, now as a function of $\mathbf{e}_{0}$ and $t_{0}$, which is described by the adjoint Smoluchowski equation (see, e.g., [29] for more details)

$$
\partial_{t_{0}} P\left(\mathbf{e}, t^{*} \mid \mathbf{e}_{0}, t_{0}\right)=-\mathcal{L}^{+}\left(\mathbf{e}_{0}\right) P\left(\mathbf{e}, t^{*} \mid \mathbf{e}_{0}, t_{0}\right),
$$

where

$$
\mathcal{L}^{+}\left(\mathbf{e}_{0}\right)=\boldsymbol{\Omega}_{\mathrm{HI}}\left(\mathbf{e}_{0}\right) \cdot \mathcal{R}\left(\mathbf{e}_{0}\right)+D_{r} \mathcal{R}^{2}\left(\mathbf{e}_{0}\right)
$$

is the adjoint operator of $\mathcal{L}\left(\mathbf{e}_{0}\right)$. In our problem $\boldsymbol{\Omega}_{\mathrm{HI}}\left(\mathbf{e}_{0}\right)=\Omega_{\mathrm{HI}}\left(\theta_{0}\right) \mathbf{e}_{\phi_{0}}$ (see main text) and so $\boldsymbol{\Omega}_{\mathrm{HI}}\left(\mathbf{e}_{0}\right)$. $\mathcal{R}\left(\mathbf{e}_{0}\right)=\Omega_{\mathrm{HI}}\left(\theta_{0}\right) \partial_{\theta_{0}}$ is independent of $\phi_{0}$. Taking now the integral $\int_{0}^{2 \pi} \mathrm{d} \phi \int_{0}^{2 \pi} \mathrm{d} \phi_{0} \ldots$ of Eq. (12) yields

$$
\partial_{t_{0}} p\left(\theta, t^{*} \mid \theta_{0}, t_{0}\right)=-\mathcal{L}^{+}\left(\theta_{0}\right) p\left(\theta, t^{*} \mid \theta_{0}, t_{0}\right)
$$

with $p\left(\theta, t^{*} \mid \theta_{0}, t_{0}\right)=\int_{0}^{2 \pi} \mathrm{d} \phi_{0} \int_{0}^{2 \pi} \mathrm{d} \phi P\left(\theta, \phi, t^{*} \mid \theta_{0}, \phi_{0}, t_{0}\right)$ and $\mathcal{L}^{+}\left(\theta_{0}\right)=\Omega_{\mathrm{HI}}\left(\theta_{0}\right) \partial_{\theta_{0}}+D_{r}\left(\partial_{\theta_{0}}^{2}+\cot \partial_{\theta_{0}}\right)$ (see main text). Finally, taking the integral $\int_{\theta^{*}}^{\pi} \ldots \sin \theta \mathrm{d} \theta$ of Eq. (14) and introducing $t=t^{*}-t_{0}$ results in Eq. (2) of the main text.

\section{Estimate of Mean Detention Time}

In the following, we derive the estimates for the mean detention times of pushers and pullers [see Eqs. (6) and (7) of main text], when hydrodynamic interactions with the surface are sufficiently large. We will consider the escape from the surface as an escape process from the minimum of an effective potential and formulate equations reminiscent of Kramers' mean escape rate [29]. The dimensionless adjoint Smoluchowski equation for $g\left(\theta^{*}, t \mid \theta_{0}\right)$ [see Eq. (2) of the main text] can be rewritten as

$$
\frac{\partial g\left(\theta^{*}, t \mid \theta_{0}\right)}{\partial t}=\left[\frac{1}{2 \mathrm{Pe}_{r}} \frac{\partial}{\partial \theta_{0}}-\frac{\partial V_{\mathrm{eff}}\left(\theta_{0}\right)}{\partial \theta_{0}}\right] \frac{\partial g\left(\theta^{*}, t \mid \theta_{0}\right)}{\partial \theta_{0}},
$$

where the effective potential $V_{\mathrm{eff}}(\theta)$ for the force-dipole swimmers reads $V_{\text {eff }}(\theta)=V+V_{r}=3 p \cos ^{2} \theta / 16-$ $\ln (\sin \theta) /\left(2 \mathrm{Pe}_{r}\right)$. The stable orientations $\theta_{s}$ of the pusher and the puller are determined by the potential minimum of $V_{\text {eff }}\left(\theta_{s}\right)$, where $\left.V_{\text {eff }}^{\prime}(\theta)\right|_{\theta=\theta_{s}}=0$ and $\left.V_{\text {eff }}^{\prime \prime}(\theta)\right|_{\theta=\theta_{s}}>0$. The stable orientation of the pusher reads $\theta_{s}=\pi / 2$ and the puller orients at $\theta_{s}=\pi-\arcsin \left[2 / \sqrt{-3 p \mathrm{Pe}_{r}}\right]$. As explained in the main text, the escape angle of the pusher is $\theta^{*}=\arccos \left[\left(-4+\sqrt{16+27 p^{2}}\right) /(9 p)\right]$ and for the puller $\theta^{*}=\pi / 2$.

In the following we assume that the angular dynamics $\theta(t)$ can be separated into two processes: First, after reaching the wall at the incoming angle $\theta_{0}$, the swimmer is oriented by hydrodynamic interactions towards the stable orientation $\theta_{s}$ at the wall. There, the swimmer orientation equilibrates fast in the minimum and a (quasi-) stationary distribution $p(\theta)$ peaked around $\theta_{s}$ is established. All in all, this takes the typical time $\tau\left(\theta_{0} \rightarrow \theta_{s}\right)$. Second, starting from $\theta_{s}$ the swimmer tries to escape from the wall. For reaching the escape angle $\theta^{*}$, it has to move up the potential difference $\Delta V_{\text {eff }}=V_{\text {eff }}\left(\theta^{*}\right)-V_{\text {eff }}\left(\theta_{s}\right)$, which takes the time $\tau\left(\theta_{s} \rightarrow \theta^{*}\right)$. Then, the mean first-passage time from the wall can be approximated by $T \approx \tau\left(\theta_{0} \rightarrow \theta_{s}\right)+\tau\left(\theta_{s} \rightarrow \theta^{*}\right)$. We now assume that $\tau\left(\theta_{s} \rightarrow \theta^{*}\right) \gg \tau\left(\theta_{0} \rightarrow \theta_{s}\right)$ and hence $T \approx \tau\left(\theta_{s} \rightarrow \theta^{*}\right)$. So, we can approximate the escape process from the surface as a quasi-stationary dissociation process to move up the potential difference $\Delta V_{\text {eff }}$ by starting at the potential minimum $\theta_{s}$.

Now, the theory of mean first-passage times provides an exact expression for the mean escape time from the wall, calculated as the mean time needed for reaching a potential value $V_{\text {eff }}\left(\theta^{*}\right)$ at $\theta^{*}$ when starting from $V_{\text {eff }}\left(\theta_{s}\right)$ at $\theta_{s}$ [29]:

$$
T=2 \mathrm{Pe}_{r} \int_{\theta^{*}}^{\theta_{s}} d \theta_{1} e^{2 \mathrm{Pe}_{r} V_{\mathrm{eff}}\left(\theta_{1}\right)}\left(\int_{\theta_{1}}^{\pi} d \theta_{2} e^{-2 \mathrm{Pe}_{r} V_{\mathrm{eff}}\left(\theta_{2}\right)}\right) .
$$

Eq. (16) cannot be solved exactly. However, for $\mathrm{Pe}_{r} \Delta V_{\text {eff }} \gg 1$ the first integral on the right-hand side of Eq. (16) is dominated by the maximum potential value at $\theta^{*}$ so that $T$ can be approximated by [29]

$$
T \approx 2 \mathrm{Pe}_{r} \int_{\theta^{*}}^{\theta_{s}} d \theta_{1} e^{2 \mathrm{Pe}_{r} V_{\mathrm{eff}}\left(\theta_{1}\right)} \int_{\theta^{*}}^{\pi} d \theta_{2} e^{-2 \mathrm{Pe}_{r} V_{\mathrm{eff}}\left(\theta_{2}\right)} .
$$

Now, the two independent integrations are mainly governed by the regions around the respective maximum values of the integrands at $\theta^{*}$ and $\theta_{s}$.

\subsection{Pusher}

For smooth potential barriers $T$ can be calculated by using Kramers' escape-time formula [29]. However, it is not applicable to the escape of a pusher from the wall. 
The reason is that the pusher does not pass a potential maximum of $V_{\text {eff }}$ but only has to reach $\theta^{*}$ at the slope of the potential, where it ultimately escapes. An approximate solution of Eq. (17) can be found by expanding the potential around $\theta^{*}$ for the $\theta_{1}$ integration and around $\theta_{s}$ for the $\theta_{2}$ integration in Eq. (17). So, integration over $\theta_{2}$ yields

$$
\begin{aligned}
& \int_{\theta^{*}}^{\pi} d \theta_{2} e^{-2 \mathrm{Pe}_{r} V_{\mathrm{eff}}\left(\theta_{2}\right)} \\
\approx & \int_{-\infty}^{\infty} d \theta_{2} e^{-2 \mathrm{Pe}_{r}\left[V_{\mathrm{eff}}\left(\theta_{s}\right)+\frac{1}{2} V_{\mathrm{eff}}^{\prime \prime}\left(\theta_{s}\right)\left(\theta_{2}-\theta_{s}\right)^{2}\right]} \\
= & e^{-2 \mathrm{Pe}_{r} V_{\mathrm{eff}}\left(\theta_{s}\right)} \sqrt{\frac{\pi}{\mathrm{Pe}_{r} V_{\mathrm{eff}}^{\prime \prime}\left(\theta_{s}\right)}}=\sqrt{\frac{2 \pi}{\frac{3}{4} p \mathrm{Pe}_{r}+1}},
\end{aligned}
$$

where we used $V_{\text {eff }}^{\prime \prime}\left(\theta_{s}\right)=3 p / 8+\left(2 \mathrm{Pe}_{r}\right)^{-1}$ and $V_{\text {eff }}\left(\theta_{s}\right)=$ 0 , and integration over $\theta_{1}$ is evaluated to

$$
\begin{gathered}
\int_{\theta^{*}}^{\theta_{s}} d \theta_{1} e^{2 \mathrm{Pe}_{r} V_{\mathrm{eff}}\left(\theta_{1}\right)} \approx \int_{\theta^{*}}^{\infty} d \theta_{1} e^{2 \mathrm{Pe}_{r}\left[V_{\mathrm{eff}}\left(\theta^{*}\right)+V_{\mathrm{eff}}^{\prime}\left(\theta^{*}\right)\left(\theta_{1}-\theta^{*}\right)\right]} \\
=\frac{e^{2 \mathrm{Pe}_{r} V_{\mathrm{eff}}\left(\theta^{*}\right)}}{2 \mathrm{Pe}_{r}\left|V_{\mathrm{eff}}^{\prime}\left(\theta^{*}\right)\right|}=\frac{1}{2 \mathrm{Pe}_{r} \Omega\left(\theta^{*}\right)} e^{2 \mathrm{Pe}_{r} \Delta V_{\mathrm{eff}}},
\end{gathered}
$$

where we used $V_{\text {eff }}^{\prime}\left(\theta^{*}\right)=-\Omega\left(\theta^{*}\right)$ (see main text) and $\Delta V_{\text {eff }}=V_{\text {eff }}\left(\theta^{*}\right)$. Hence, with Eqs. (17), (18) and (19) we obtain Eq. (6) of the main text.

\subsection{Puller}

The escape of the puller $(p<0)$ at the location $\theta^{*}=$ $\pi / 2$ of the potential maximum $U\left(\theta^{*}\right)=0$ starting from the potential minimum $U\left(\theta_{s}\right)<0$ can be calculated by using a harmonic expansion around both the potential minimum and the potential maximum. So, the integrals in Eq. (17) can be approximated by

$$
\begin{aligned}
& \int_{\theta^{*}}^{\pi} d \theta_{2} e^{-2 \mathrm{Pe}_{r} V_{\mathrm{eff}}\left(\theta_{2}\right)} \\
\approx & \int_{-\infty}^{\infty} d \theta_{2} e^{-2 \mathrm{Pe}_{r}\left[V_{\mathrm{eff}}\left(\theta_{s}\right)+\frac{1}{2} V_{\mathrm{eff}}^{\prime \prime}\left(\theta_{s}\right)\left(\theta_{2}-\theta_{s}\right)^{2}\right]} \\
= & \frac{\sqrt{\pi} e^{-2 \mathrm{Pe}_{r} V_{\mathrm{eff}}\left(\theta_{s}\right)}}{\sqrt{\mathrm{Pe}_{r} V_{\mathrm{eff}}^{\prime \prime}\left(\theta_{s}\right)}}=\sqrt{\frac{\pi}{\frac{3}{4}|p| \mathrm{Pe}_{r}-1}} e^{2 \mathrm{Pe}_{r} \Delta V_{\mathrm{eff}}},
\end{aligned}
$$

where we used $\Delta V_{\text {eff }}=\left|V_{\text {eff }}\left(\theta_{s}\right)\right|$ and $V_{\text {eff }}^{\prime \prime}\left(\theta_{s}\right)=-3 p / 4-$ $1 / \mathrm{Pe}_{r}$, and by

$$
\begin{aligned}
& \int_{\theta^{*}}^{\theta_{s}} d \theta_{1} e^{2 \mathrm{Pe}_{r} V_{\mathrm{eff}}\left(\theta_{1}\right)} \\
\approx & \int_{\theta^{*}}^{\infty} d \theta_{1} e^{2 \mathrm{Pe}_{r}\left[V_{\mathrm{eff}}\left(\theta^{*}\right)+\frac{1}{2} V_{\mathrm{eff}}^{\prime \prime}\left(\theta^{*}\right)\left(\theta_{1}-\theta^{*}\right)^{2}\right]} \\
= & \frac{1}{2} \sqrt{\frac{\pi}{\mathrm{Pe}_{r}\left|V_{\mathrm{eff}}^{\prime \prime}\left(\theta^{*}\right)\right|}} e^{2 \mathrm{Pe}_{r} V_{\mathrm{eff}}\left(\theta^{*}\right)}=\frac{1}{2} \sqrt{\frac{2 \pi}{\frac{3}{4}|p| \mathrm{Pe}_{r}-1}}
\end{aligned}
$$

where we used $V_{\text {eff }}^{\prime \prime}\left(\theta^{*}\right)=3 p / 8+1 /\left(2 \mathrm{Pe}_{r}\right)$ and $V_{\text {eff }}\left(\theta^{*}\right)=$ 0. Hence, with Eqs. (17), (20) and (21) we obtain Eq. (7) of the main text.

We note that in the limit $|p| \mathrm{Pe}_{r} \rightarrow \infty$ the potential around the minimum at $\theta_{s} \rightarrow \pi$ is highly asymmetric and the quadratic expansion is not a good approximation. For this case we assume that the stable position is $\theta_{s} \approx \pi$. Then, we approximate the $\theta_{2}$-integration in Eq. (17) for the puller by

$$
\begin{aligned}
& \int_{-\infty}^{\theta_{s}} d \theta_{2} e^{-2 \mathrm{Pe}_{r} V_{\mathrm{eff}}\left(\theta_{2}\right)}=\int_{-\infty}^{\theta_{s}} d \theta_{2} e^{-2 \mathrm{Pe}_{r} V\left(\theta_{2}\right)} e^{-2 \mathrm{Pe}_{r} V_{r}\left(\theta_{2}\right)} \\
\approx & e^{-2 \mathrm{Pe}_{r} V\left(\theta_{s}\right)} \int_{-\infty}^{\theta_{s}} d \theta_{2} \sin \theta_{2} e^{-\mathrm{Pe}_{r} V^{\prime \prime}\left(\theta_{s}\right)\left(\theta_{2}-\theta_{s}\right)^{2}} \\
\approx & -e^{-2 \mathrm{Pe}_{r} V\left(\theta_{s}\right)} \int_{-\infty}^{\theta_{s}} d \theta_{2}\left(\theta_{2}-\theta_{s}\right) e^{-\mathrm{Pe}_{r} V^{\prime \prime}\left(\theta_{s}\right)\left(\theta_{2}-\theta_{s}\right)^{2}} \\
= & \frac{e^{-2 \mathrm{Pe}_{r} V\left(\theta_{s}\right)}}{2 \mathrm{Pe}_{r} V^{\prime \prime}\left(\theta_{s}\right)}=\frac{4 e^{2 \mathrm{Pe}_{r} \Delta V}}{3|p| \mathrm{Pe}_{r}}
\end{aligned}
$$

and together with Eq. (21) we obtain

$$
T\left(\theta_{s} \rightarrow \theta^{*}\right) \approx \frac{1}{2} \frac{\sqrt{\pi} e^{2 \mathrm{Pe}_{r} \Delta V}}{\sqrt{\mathrm{Pe}_{r}\left|V^{\prime \prime}\left(\theta^{*}\right)\right|} V^{\prime \prime}\left(\theta_{s}\right)}
$$

with $V^{\prime \prime}\left(\theta^{*}\right)=\frac{3 p}{8}$ and $V^{\prime \prime}\left(\theta_{s}\right)=-\frac{3 p}{8}$.

\subsection{Deterministic Detention Times}

For the deterministic source-dipole swimmer $\Omega_{\mathrm{HI}}=$ $d \theta / d t=-\frac{3 q}{8} \sin \theta$ in dimensionless units. After integration we obtain for an incoming angle $\theta_{0}$ and an escape angle $\theta^{*}=\pi / 2$ the deterministic wall detention time

$$
T^{\mathrm{det}}=\frac{8}{3 q} \ln \left(\tan \frac{\theta_{0}}{2}\right) .
$$

For a neutral squirmer close to a wall $\Omega_{\mathrm{HI}}=-M / \gamma_{r}=$ $-\frac{6 \pi}{5} \frac{\ln \epsilon^{-1}-c}{\gamma_{r}(\epsilon)} \sin \theta$ where we use $M$ from Eq. (8) of the main text and $\gamma_{r} \approx 8 \pi\left[(2 / 5) \ln \epsilon^{-1}+0.37\right]$ is the dimensionless rotational friction constant of a sphere near a wall [45]. Hence

$$
T^{\mathrm{det}}=\frac{8}{3} \frac{\left(\ln \epsilon^{-1}+0.925\right)}{\left(\ln \epsilon^{-1}-c\right)} \ln \left(\tan \frac{\theta_{0}}{2}\right) .
$$

The noiseless pusher and puller have stable orientations at the wall and hence $T^{\text {det }} \rightarrow \infty$.

\section{Additional Information on the Motion of a Swimmer Near a Wall}

\subsection{Trajectory in Front of a Wall}

Swimmer trajectories can be obtained by numerically solving Eqs. (1) of the main text. In Fig. 5] we show an 


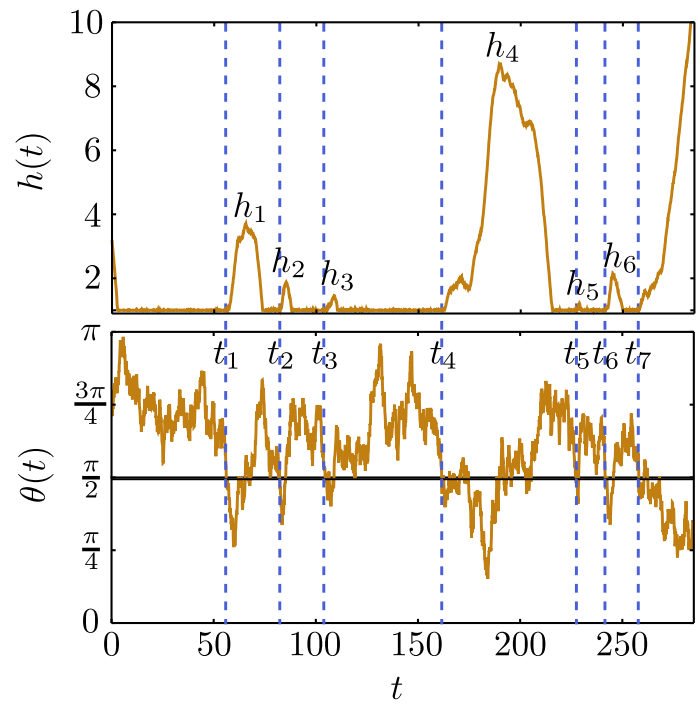

FIG. 5. Typical trajectory of a swimmer close to a wall. The time evolution of the swimmer-wall distance $h(t)$ and the orientation $\theta(t)$ is shown here for an active Brownian particle with $\mathrm{Pe}_{r}=25, \mathrm{Pe}=1000$. At times $t_{n}$ the swimmer reaches the escape angle $\theta^{*}$. Between two encounters the swimmer approaches a maximum distance $h_{n}$ above the wall.

example for an $\mathrm{ABP}$ (with $\mathrm{Pe}_{r}=25, \mathrm{Pe}=1000$ ). The swimmer starts at an angle $\theta \approx 3 \pi / 4$ at a distance $h \approx$ 3 from the wall, approaches the wall, reorients, and its position $h(t)$ fluctuates. When the swimmer reaches an escape angle $\theta^{*}=\pi / 2$ at times $t_{n}, n=1, \ldots, 7$, it swims away from the wall. Due to the persistent random walk of the swimmer it will come back to the wall infinitely many times. We only show the trajectory here until $t \approx 280$. The maximum heights above the surface $h_{n}, n=1, \ldots, 6$, between two encounters with a wall strongly fluctuate.

Hence, defining the escape of a swimmer from a surface by introducing a specific escape height $h^{*}$, strongly depends on the value of $h^{*}$. A much clearer and unambiguous definition of the escape process is our approach in the main text, where we introduce an escape angle within a $1 \mathrm{D}$ model. The resulting mean detention time is an appropriate means to compare the swimmer-wall encounters of different swimmer types to each other.

\subsection{Orientational distribution at escape height $h^{*}>1$}

One may define the escape of the swimmer from the wall by reaching a certain height $h^{*}>1$ above the wall after leaving the wall, as suggested in our 2D model [Eq.(9) in the main text]. Then the angles $\theta^{*}$ at $h^{*}$ are typically smaller than the escape angles defined in the 1D model (see main text) and are distributed over a range of $\theta^{*}$ values. Some distributions $p\left(\theta^{*} \mid h^{*}\right)$ are shown in Fig. 6 for different swimmer types for $\mathrm{Pe}_{r}=25, \mathrm{Pe}=1000$. The widths of the distributions typically increase and the mean values $\left\langle\theta^{*}\right\rangle$ decrease with increasing $h^{*}$, since

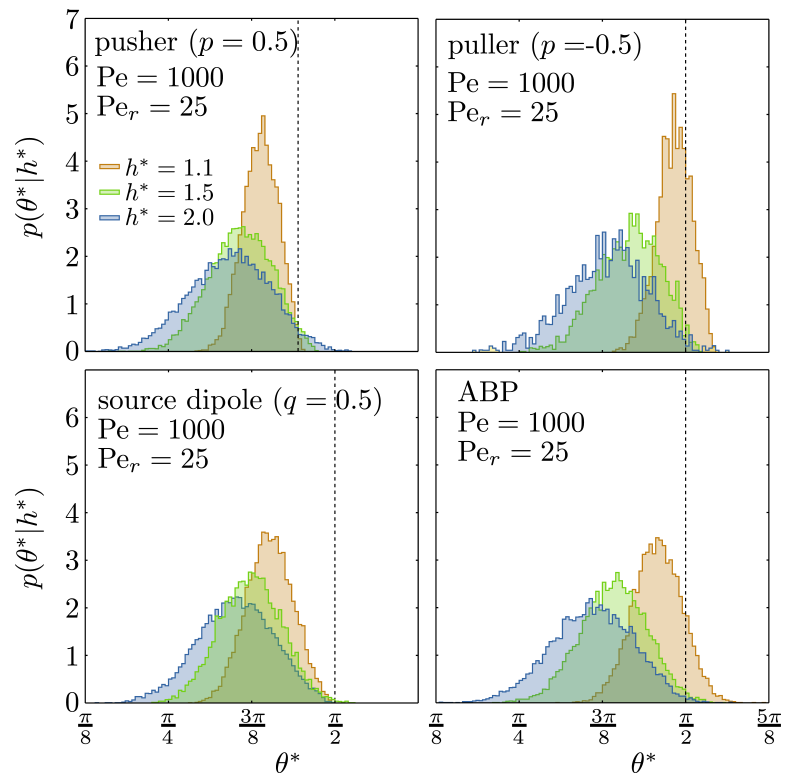

FIG. 6. Distribution of angles $\theta^{*}$ after collision with a surface for different swimmer types measured at several distances $h^{*}$ above the wall. The dashed lines show the escape angles from the $1 \mathrm{D}$ model.

the swimmers have more time to explore a larger range of orientations. Since swimmers can also reach the escape height $h^{*}$ by translational Brownian motion, the corresponding orientation angle $\theta^{*}$ can also be larger than the escape angle needed in the 1D model (dashed lines). Nevertheless, for sufficiently large $h^{*}$ escape through translational diffusion becomes negligible, as also shown in Sec. 3.3 and 3.4.

\subsection{Translational vs. Orientational Escape Times}

When a swimmer approaches a wall, it is subjected to both translational and rotational noise, quantified by the Péclet number $\mathrm{Pe}$ and the persistence number $\mathrm{Pe}_{r}$ (see definitions in the main text). For a spherical swimmer with pure thermal noise, translational and rotation diffusion constants are coupled [26] and $\mathrm{Pe}=3 \mathrm{Pe}_{r}$. For real microswimmers intrinsic noise can enhance rotational diffusion and thereby lower its persistence, such that

$$
\mathrm{Pe} \geq 3 \mathrm{Pe}_{r} .
$$

In the main text we presented the mean detention times $T$ at the wall by assuming the swimmer stays at the wall $(h \approx R)$ until it reaches the escape angle $\theta^{*}$. In the following we show that our model swimmers indeed stay close to the wall during time $T$ and despite their translational diffusion. We estimate a translational escape time $T_{t}(\Delta h)$ for reaching a height $\Delta h$ (in units of the swimmer radius $R$ ) above the surface. By setting $T_{t}=T$, we calculate the height $\Delta h$, the swimmer can reach in time $T$. We find $\Delta h<R$, so the swimmer stays close to the 
surface.

Close to the wall the swimmer velocity along the surface normal in units of the swimmer velocity $U$ is $v_{W}=$ $\cos \theta+v_{\mathrm{HI}}(\theta, h)$ due to active motion and hydrodynamic swimmer-wall interactions. For a constant drift velocity $v_{W}<0$ and a given Péclet number, one can readily solve Eq. (9) from the main text following Ref. [57]. In particular, the mean time $T_{t}$ to move away a distance $\Delta h$ from the wall after starting at the wall reads in dimensionless units

$$
T_{t}\left(\Delta h, v_{W}, \mathrm{Pe}\right)=\frac{\exp \left(2 \mathrm{Pe} \Delta h\left|v_{W}\right|\right)-1}{2 \mathrm{Pe} v_{W}^{2}}-\frac{\Delta h}{\left|v_{W}\right|} .
$$

For the limiting cases $\mathrm{Pe} \rightarrow \infty$ and $\mathrm{Pe} \rightarrow 0$ one obtains the expressions

$$
\begin{aligned}
T_{t}(\mathrm{Pe} \rightarrow \infty) & =\frac{\exp \left(2 \mathrm{Pe} \Delta h\left|v_{W}\right|\right)}{2 \mathrm{Pe} v_{W}^{2}}, \\
T_{t}(\mathrm{Pe} \rightarrow 0) & =\mathrm{Pe} \Delta h^{2} .
\end{aligned}
$$

We will use Eqs. (27) and (28) in the following to estimate lower limits for the time $T_{t}$ and hence the maximum distance $\Delta h$, which a swimmer reaches during reorienting at the wall.

\subsubsection{Active Brownian Particle (ABP)}

By rotational diffusion an $\mathrm{ABP}$ without hydrodynamic swimmer-wall interactions needs $T^{\mathrm{ABP}}<2 \mathrm{Pe}_{r}$ to reach the escape orientation for any incoming angle $\theta_{0}$, as shown in Eq. (5) of the main text. On the other hand, Eq. (27) in the limit $v_{w} \rightarrow 0$, which means parallel orientation, predicts a lower limit for $T_{t}$ to reach a height $\Delta h$ above the surface: $T_{t}^{\mathrm{ABP}}>\operatorname{Pe} \Delta h^{2}$. By setting $T \sim T_{t}$, we estimate an upper limit for the height $\left(\Delta h^{\mathrm{ABP}}<\sqrt{2 / 3}\right)$ the ABP reaches by translational diffusion during reorientation at the wall. Hence, the escape process for the $\mathrm{ABP}$ is determined by rotational diffusion at all Péclet numbers. Since for the ABP the reorientation rate does not depend on $h$, the detention times are not modified by translational diffusion.

\subsubsection{Pusher}

Figure 3 in the main text shows the detention times $T$ for force dipole swimmers depending on the dipole strength $p$ and the persistence number $\mathrm{Pe}_{r}$. For the pusher $(p>0)$ in region I we have $T<T^{\mathrm{ABP}}$ (see main text). To obtain estimates for the translational escape times $T_{t}$, we use for the velocity along the wall normal

$$
v_{W}=\cos \theta+\frac{3 p\left(3 \cos ^{2} \theta-1\right)}{8 h^{2}},
$$

where we included $v_{\mathrm{HI}}$ for a force-dipole swimmer [15]. Since at the wall $v_{W}<0$ for the pusher [Eq. (29)], $T_{t}>$ $T_{t}^{\mathrm{ABP}}$. So, the region-I pusher reaches an even smaller height than the $\mathrm{ABP}\left(\Delta h<\Delta h^{\mathrm{ABP}}\right)$.
In region II (large $\mathrm{Pe}_{r}$ and large $p$ ) the mean detention time is $T \sim \exp \left(3 \mathrm{Pe}_{r} p \cos ^{2} \theta^{*} / 8\right)$ (see Eq. (6) of main text) with $\cos \theta^{*}=\left(-4+\sqrt{16+27 p^{2}}\right) /(9 p)$. On the other hand, for distances up to $\Delta h$ we have the lower limit $\left|v_{\mathrm{HI}}\right|>3 p /\left[8(\Delta h+1)^{2}\right]=$ const [Eq. (29)] $]$ and we use Eq. (27) to obtain a lower bound for $T_{t}$. In the limit $\mathrm{Pe} \rightarrow \infty$ [which applies in region II due to Eq. [(26)], the pusher needs the time $T_{t} \gtrsim \exp \left[(3 / 4) p \operatorname{Pe} \Delta h /(\Delta h+1)^{2}\right]$ [see Eq. (28)] to reach a distance $\Delta h$ from the wall with the stable orientation $\theta_{s}=\pi / 2$. Hence, by comparing $T_{t}$ with $T$, we obtain $\Delta h /(\Delta h+1)^{2}<(-4+$ $\left.\sqrt{16+27 p^{2}}\right)^{2} /\left(486 p^{2}\right)$, and $\Delta h<0.063$ for all $p$.

So, for pushers in region I and region II the escape process is always determined by rotational and not translational motion. However, fluctuations in $h$ may alter the detention times due to the $h$-dependent hydrodynamic swimmer-wall interactions, as shown in Sec. 3.4.

\subsubsection{Puller}

A puller $(p<0)$ with sufficiently large $|p|$ and $\mathrm{Pe}_{r}$ has a stable orientation $\theta_{s} \approx \pi$ at the wall, and its mean detention time is $T \sim \exp \left(3|p| \mathrm{Pe}_{r} / 8\right)$ (Eq. (7) of the main text). By using Eq. (28) and Eq. (29), the time to reach a height $\Delta h$ via translational diffusion is $T_{t} \gtrsim \exp \left[2 \mathrm{Pe} \Delta h\left(1+(3 / 4)|p| /(\Delta h+1)^{2}\right)\right]$ since $\left|v_{\mathrm{HI}}\right| \geq 1+(3 / 4)|p| /(\Delta h+1)^{2}$ [see Eq. (29)]. Comparing now $T_{t}$ and $T$, and using Eq. (26), we obtain $(3 / 8)|p|<6 \Delta h\left(1+(3|p|) /\left(4(\Delta h+1)^{2}\right)\right)$, which results in $\Delta h<0.1$.

For a puller with small $|p| \mathrm{Pe}_{r}$ the detention time $T \approx T^{\mathrm{ABP}}<2 \mathrm{Pe}_{r}$. Since $v_{W}<0$ around the stable orientation, $T_{t}>T_{t}^{A B P}$ and $\Delta h<\sqrt{2 / 3}$.

So, also a puller is not able to diffuse far from the wall although, similar to the pusher, fluctuations in $h$ may influence the detention times as shown in Sec. 3.4.

\subsubsection{Source Dipole Swimmer}

The source dipole swimmer reorients faster than the $\mathrm{ABP}$ at the wall towards the escape angle $\left(T<T^{\mathrm{ABP}}\right)$. The velocity along the wall normal reads $v_{W}=\cos \theta-$ $q \cos \theta / h^{3}<0$ [15] for orientations towards the wall such that $T_{t}>\mathrm{Pe} \Delta h^{2}$ [Eqs. (27)] for any Péclet number, and $\Delta h<\sqrt{2 / 3}$. So, also the source dipole swimmer does not leave the wall via translational diffusion.

\subsection{Comparison of $1 D$ and 2D Model}

In the main text we introduced a 1D model [Eq. (2) of main text] for calculating distributions of the swimmerwall detention times but also outlined a 2D model [Eq. (9) of main text]. While in the 1D model the escape from the surface is defined by reaching an escape angle $\theta^{*}$, in the $2 \mathrm{D}$ model the swimmer escapes when it reaches a specific height $h^{*}$ above the wall. In Fig. 7(a-c) we now 

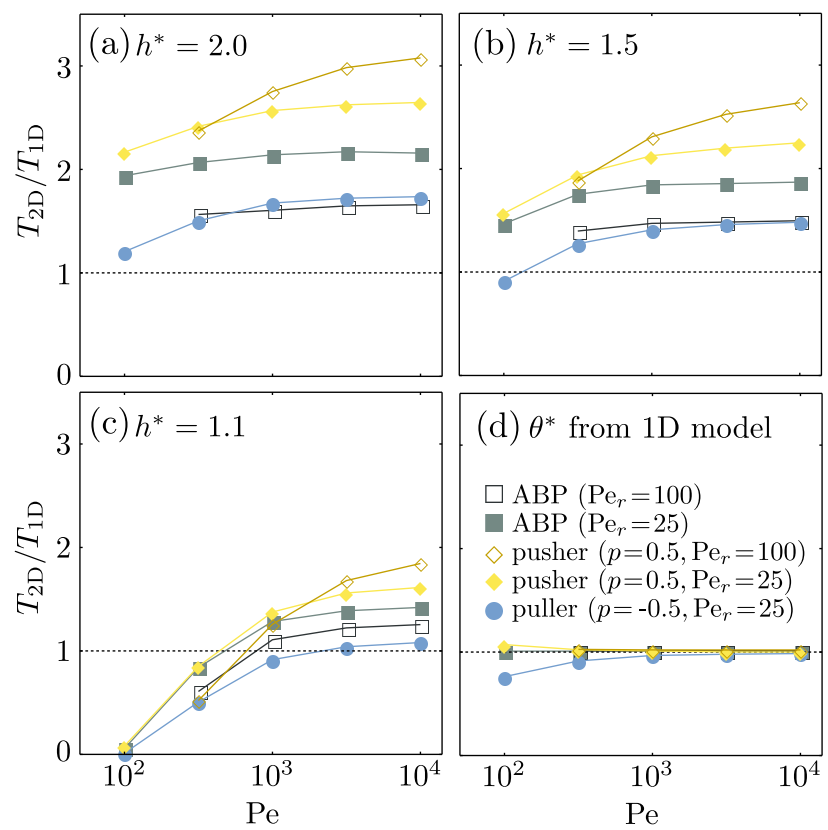

FIG. 7. (a-c) Comparison of 2D model with 1D model for the incoming angle $\theta_{0}=3 \pi / 4$ for different escape heights $h^{*}$. In (d) the mean detention time for reaching the escape angle $\theta^{*}$ in the full $2 \mathrm{D}$ model is compared to the $1 \mathrm{D}$ model.

compare the mean detention times determined from both models for different swimmer types, persistence numbers, Péclet numbers, and escape distances $h^{*}=1+\Delta h$ for the incoming angle $\theta_{0}=3 \pi / 4$. We observe that for small heights and sufficiently small $\mathrm{Pe}, T_{2 \mathrm{D}}<T_{1 \mathrm{D}}$ since during reorientation at the wall the swimmers can reach $h^{*}$ via translational diffusion. For sufficiently large $h^{*}$, one always finds $T_{2 \mathrm{D}}>T_{1 \mathrm{D}}$, since the swimmer needs additional time to reach $h^{*}$ after leaving the surface.

Reorientation rates due to hydrodynamic swimmerwall interactions, $\Omega_{\mathrm{HI}}$, depend in general on the distance from the wall [15]. Hence, fluctuations in the position $h$ above the surface may influence the detention times for reaching the escape angle $\theta^{*}$ defined in the $1 \mathrm{D}$ model. In Fig. 7(d) we show the mean detention time $T_{2 \mathrm{D}}$ by solving the full Langevin dynamics for an incoming angle $\theta_{0}$ and compare it to $T_{1 \mathrm{D}}$ from the $1 \mathrm{D}$ model. Depending on the specific swimmer type, translational noise can either enhance or decrease the detention times for sufficiently small Pe. However, the effect of translational noise is in general rather small. As expected, for active Brownian particles with $\Omega_{\mathrm{HI}}=0$ the detention times are independent of translational noise.

\section{Effect of Steric Interactions for "Chlamy- domonas"}

Our model is able to treat steric swimmer-wall interactions, which can occur for any swimmer with flagellawall contact. As an example we show DTDs of a sim-

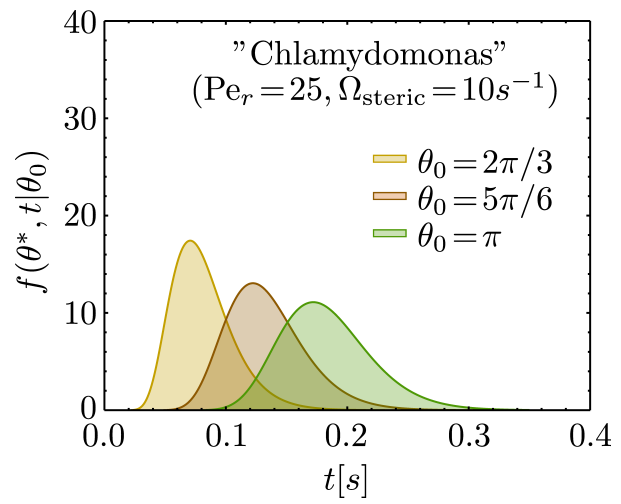

FIG. 8. Detention time distributions $f\left(\theta^{*}, t \mid \theta_{0}\right)$ for a simple Chlamydomonas model $\left(\mathrm{Pe}_{r}=25, \Omega_{\text {steric }}=10 s^{-1}, \theta^{*}=\right.$ $0.64 \pi)$ for different incoming angles $\theta_{0}$.

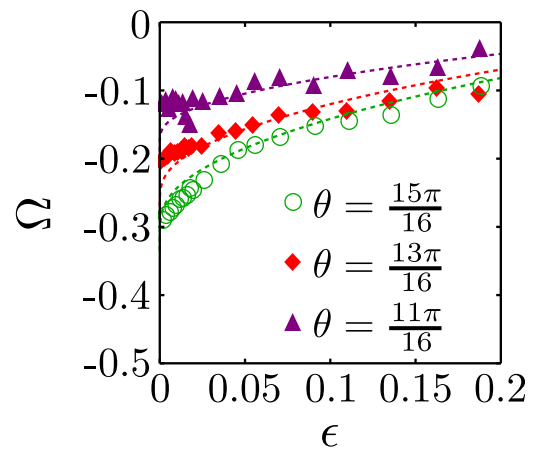

FIG. 9. Distance-dependent angular velocity of a neutral squirmer in front of a no-slip wall for several orientations $\theta$ compared to lubrication theory (dashed lines).

plified model for Chlamydomonas. We neglect the hydrodynamic swimmer-wall interactions and simplify the time-dependent flagella-wall interactions by assuming a constant reorientation rate $\Omega_{\text {steric }}>0$, which rotates the cell away from the wall as discussed in 12]. In our 1D model we use realistic values for the persistence number $\left(\mathrm{Pe}_{r}=25\right)$, a reorientation rate $\left(\Omega_{\text {steric }}=10 \mathrm{rad} / s\right)$, and an escape angle $\left(\theta^{*}=0.64 \pi\right)$ as suggested in Ref. [12]. Typical DTDs for different incoming angles $\theta_{0}$ are shown in Fig. 8. In accordance with Ref. [12], the detention time of a Chlamydomonas swimmer at the wall is always less than half a second.

\section{MPCD Simulations}

We perform MPCD simulations by using the same parameters to model the fluid and the squirmer as in [53]. To determine the constant $c$ of Eq. (8) in the main text, we measure the wall-induced angular velocity $\Omega(\epsilon, \theta)$ of a large number of neutral squirmers in front of a no-slip wall in separate simulations to average out thermal noise. Figure 9 shows $\Omega(\epsilon)$ for different $\theta$ compared to the analytic expression obtained by using Eq. (8) of the main 
text and $\Omega=-M / \gamma_{r}$. The curves fit best for $c \approx 0.9$.

We then perform simulations of squirmer-wall interac- tions for different incoming angles $\theta_{0}$ and measure the detention times at the wall. The mean distance from the wall averaged over all trajectories is $\epsilon \approx 0.01$. 